\title{
Validated novel software to measure the conspicuity index of lesions in DICOM images
}

\author{
K. R. Szczepura, D. J. Manning
}

University of Salford, Manchester, UK

\begin{abstract}
\section{Description of purpose}

A novel software programme and associated Excel spreadsheet has been developed to provide an objective measure of the expected visual detectability of focal abnormalities within DICOM images.
\end{abstract}

\section{Methodology}

ROIs are drawn around the abnormality, the software then fits the lesion using a least squares method to recognise the edges of the lesion based on the full width half maximum. 180 line profiles are then plotted around the lesion, giving 360 edge profiles.

The co-ordinates show in Figure 1 are captured, as well the standard deviation of the pixel values within the background and lesion (representing anatomical noise and lesion noise respectively).

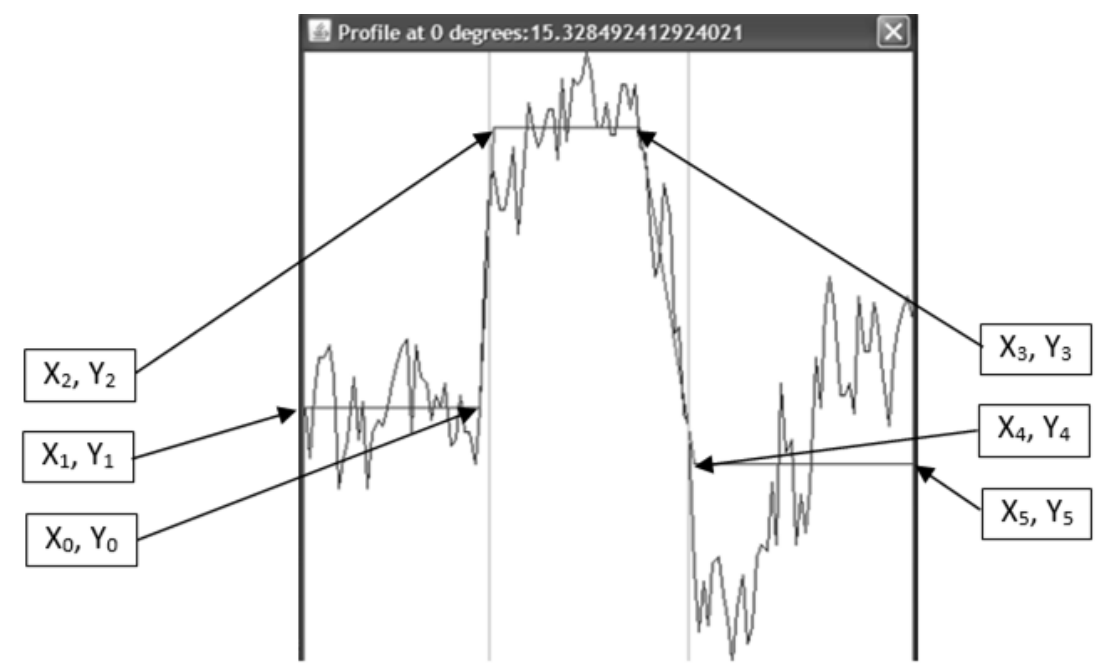

An Excel spreadsheet has been developed to allow variables to be calculated, including SNR and CNR. A conspicuity index has also been developed: 
$\chi=\frac{d \tan [\theta-1] \Delta G L}{\sqrt{\sigma_{s}^{2}+\sigma_{n}^{2}}}$

Where:

$\chi \quad=$ conspicuity index

$d \quad=$ lesion dimension $\left(\mathrm{X}_{3}-\mathrm{X}_{2}\right)$

$\theta \quad=$ maximum slope angle of all the line profiles

For each side of the line profile:

$\theta=\tan ^{-1}\left[\frac{Y_{1}-Y_{0}}{X_{1}-X_{0}}\right]$

$\theta=\tan ^{-1}\left[\frac{Y_{1}-Y_{0}}{X_{1}-X_{0}}\right]$

$\Delta G L=Y_{2}-\frac{\left(Y_{1}+Y_{4}\right)}{2}=$ the difference in average grey levels between the lesion and the background

$\sigma_{s}=s d T o p$

$\sigma_{n}=\frac{s d 1+s d 2}{2}$

\section{Results}

The software has been validated using the GAMMEX ACR CT accreditation phantom, varying mA, $\mathrm{kVp}$ and slice thickness (ST) and the results have been found to give a linear response:
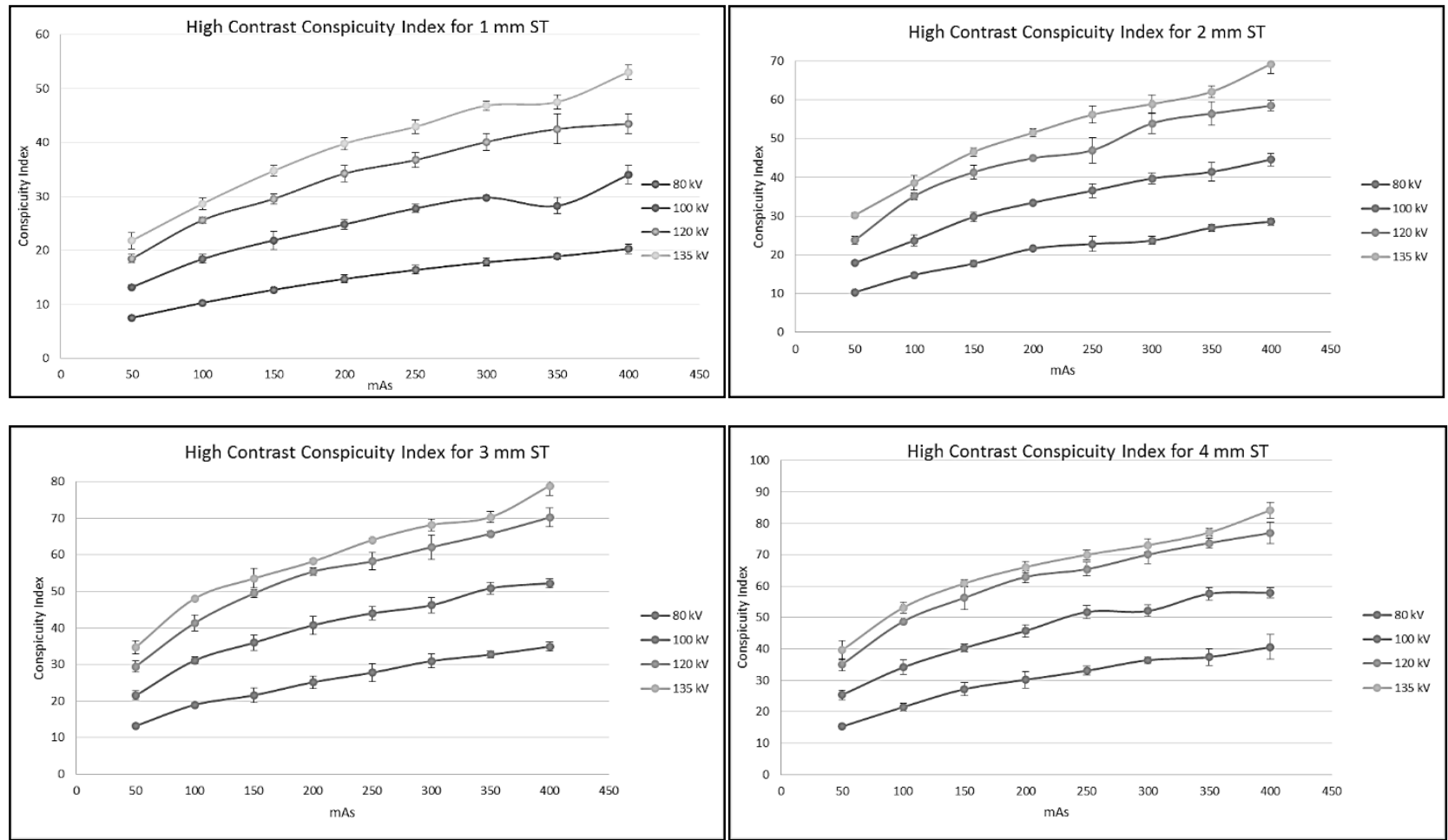

\section{Conclusion}

A novel software programme has been validated to allow calculation of many physical properties of lesions. Additionally, a new measure of conspicuity index has been developed for focal lesions.

The analysis could be further developed to incorporate reader decision-analysis data and eye-tracking data allowing correlations between physical and perception measures to be made beyond basic CNR calculations. It could also be used as a tool to distinguish between perceptual and cognitive error. 
Further refinements could lead to measures of the detectability of more diffuse disease features.

Keywords: objective, conspicuity, index, image quality

\section{INTRODUCTION}

It is well known that the performance of people reporting medical images is not perfect, both false positive and false negative decisions can be made, which can impact on the patient pathway. Errors such as these can be put down to two main factors, poor lesion detectability due to the imaging process, and cognitive or perceptual errors.

The quality of an image can be determined in numerous ways. Medical imaging systems are maintained and quality assured using structured phantoms, and measures such as signal to noise ratio (SNR) contrast to noise ratio (CNR), spatial resolution in terms of modulation transfer function (MTF) [1,2] are used to ensure the system is still functioning within expected limits. It needs to be recognised however, that these measures, although essential for maintaining and assuring standards of equipment, do not represent the complexities of a diagnostic image[1] and their outcome measure is not directly related to any specific radiological task. As well as the natural tissue variations within patients, quality control measures are taken under strict protocols, positioning and acquisition parameters, which do not represent the acquisition of an image of a patient. The term lesion conspicuity is an attempt to represent the visibility of a lesion taking into consideration the structure of the lesion, and the tissues surrounding it as well as taking into account the diagnostic potential of the image for a well-defined radiological task.

\section{Conspicuity}

The term 'conspicuity' was first used in 1974 by Revesz et al[3], and defined as the lesion contrast divided by the surrounding complexity, where the lesion contrast is the density change across the lesion border, and the surround complexity is the rate of fluctuation of the density around the lesion border. This led to a definition of structural, or anatomical, noise which takes into account the surrounding structures and artefacts. However is was recognised within this seminal piece of work that this is a simple equation and does not take into account other features that could affect the conspicuity of a lesion.

\section{Factors affecting conspicuity}

An important consideration to take into account is that a diagnostic radiograph is not a simple shadow of the anatomy, it is a complex summation of a polyenergtic beam of X-rays that have interacted in a $3 \mathrm{D}$ object within multiple layers. Therefore, as it is a summation of several layers, subtle lesions may be obscured by overlying anatomical structures[4]. Samei et al[5] inserted simulated lesions at different positions on a chest radiographs and found significant variation in lesion detectibility as a function of position, finding that local anatomic variations surrounding and overlying subtle lesions on a chest radiograph that are created by the projection of anatomic features in the thorax, such as ribs and pulmonary vessels, can greatly influence the detection of lesions.

Even in 3D imaging such as CT, adjacent anatomy can obscure lesions. Li et al[6] undertook a review of missed cancers during CT screening using low dose helical CT in a general population, and found that all 32 missed lesions were found to be in the interpulmonary region, and that of the 23 missed lesions due to detection (rather than interpretation) error, 19 of those were due to the fact that the lesions appearance was similar to that of normal structures. When measuring signal to noise or contrast to noise ratio, usually the noise is measured from a background that doesn't include complex surrounding structures, therefore these complexities that affect the detectability of a lesion are not recognised with these standard measures.

Noise is defined as unwanted information on the image[1], and there are two types that need to be taken into account when considering the conspicuity of the lesion: the structural noise and the radiographic noise. Structural noise, as discussed above, is task dependant. However radiographic noise is not dependant on the subject being imaged, but is stochastic in nature and dependent on many factors, such as the exposure factors used and the capability of the 
detector[1]. Radiographic noise is measured using SNR, and provides information about the system capability, but does not give the full information about the noise within a clinical image.

The size of a focal lesion is an important factor in determining its conspicuity. Many studies have found that lesions that are smaller than $1 \mathrm{~cm}$ are missed when viewing $2 \mathrm{D}$ images[7], and $3 \mathrm{~mm}$ is deemed the threshold size limit for detecting a lesion[4]. In mammography Birdwell et al[8] found that $81 \%$ of missed lesions in their study were found to be less than $20 \mathrm{~mm}$ even when retrospectively assessed, and Michealson et al[9] found that the median size of lesions to be detectable by mammographic screening was $7 \mathrm{~mm}$, with only $40 \%$ of lesions being seen at $5 \mathrm{~mm}$. As previously discussed, Li et al[6] found that in CT 11 of the 32 missed lesions could not be identified as they were barely discernible due to their size $(<2 \mathrm{~mm})$. It is recognised, however, that even the smallest image feature can be detected if its contrast against the background is high enough, and this principle is used to good effect in the contrast/detail test objects in quality assurance programmes [33].

Contrast is an essential measure of the diagnostic capability of a system. CNR and SNR are standard measures used to represent this, in clinical assessment of images as well as in quality control. They are both size independent if they are used in isolation as quantitative measures of image quality. They represent the difference in signal amplitude between the lesion or test feature and the background $[2,10]$. Both SNR and CNR are used as an objective measure of the quality of an image. Measures are taken of the signal of the lesion, and the signal of the surrounding areas and this data is used to calculate either the SNR or the CNR. Contrast agents such as barium sulphate in fluoroscopy, iodine in CT etc. can be added to improve visibility of the structures of interest. Improved contrast leads to greater detectability of a lesion, and so is an important measure of the image quality capabilities of a system.

The sharpness refers to the ability of the system to represent distinct anatomical features within the object being imaged[1], therefore the sharpness of the border of a lesion impacts on its visibility. There are mathematical techniques to measure the sharpness of a system. Point and line spread functions and their Fourier transform, the MTF all measure the resolving capability[1] or spatial resolution of a system, and take into account issues such as focal spot size, the polyenergetic beam, and any magnification that can cause blurring of edges. Blurring can also occur due to voluntary or involuntary patient motion. Blurring causes reduced visibility of details, image un-sharpness and reduced spatial resolution. The $3 \mathrm{~mm}$ minimum size of lesion visible, as discussed above is only applicable if the edges of the structure are parallel to the X-ray beam, if the margins are bevelled (either due to blur, or anatomical causes) then this influences the visible threshold size[4]. Edge sharpness has a powerful influence on the probability that an image feature will be detected. This is because loss of spatial resolution reduces the effectiveness of the Mach band phenomenon in the visual system which so enhances narrow gradients between adjacent regions of different grey-level [11].

In summary, the factors that have been reported to affect the conspicuity of a lesion have been found to be the structural noise within and surrounding a lesion, the size of the lesion, the contrast and the sharpness of the edges.

\section{Measuring Conspicuity}

Likert scales are often used in observer studies in attempts to quantify conspicuity [12-18], when comparing modalities, varying acquisition factors, or using different contrast agents. The likert scales used vary greatly within these studies, in terms of number of points on the scale, and the terms used for each of the points, and the scale is always task dependant. However, the use of likert scales are open to bias[19], because both perceptual and cognitive errors may be included within the task, therefore, the use of likert scales is not an objective measure of conspicuity.

Conspicuity has also been assessed using the two alternate forced choice method, 2AFC are those where the participant is asked to choose between two images, one is a static reference image the other varies depending on the research question being asked. Due to the way the studies are designed, these techniques are less susceptible to bias than likert scales and present low variability. However large sets of images are needed and it can be time consuming both in terms of the individual observer, and the number of observers required in the study, making it a costly technique. It needs to be noted however that numerous questions can be asked of the observer, not just the conspicuity, during 2AFC, adding to the complexity depth of the data obtained. An issue to consider with $2 \mathrm{AFC}$ is that it provides ordinal data, where the images are sorted in order, and the difference between the ranks are assumed to be equal, therefore the findings are dependent on the dataset used within the study. 
Various studies have used region of interest data to measure conspicuity[20, 21] comparing the lesion to the surrounding tissue, and although this is an objective measure that indicates the conspicuity of the lesion it does not take into account the other factors that affect the conspicuity, such as lesion size and sharpness as discussed above.

Manning et al[22] first proposed a method of combining the factors that describe conspicuity into a single equation. This equation took into account those factors that impact on conspicuity of a lesion based on the saliency of the image feature to the visual system, Line profiles were plotted across a lesion, extending into the immediate surrounding background and the following equation was developed.

$$
\chi=\frac{d \tan [\theta-1] \Delta G L}{\sqrt{\sigma_{s}^{2}+\sigma_{n}^{2}}}
$$

Equation 1 calculation of conspicuity index[22]

Where:

$\chi=$ conspicuity index

$\mathrm{d}=$ maximum lesion dimension

$\theta=$ maximum edge angle

$\Delta \mathrm{GL}=$ mean contrast (difference in grey level)

$\sigma_{\mathrm{s}}=$ mean noise within the lesion

$\sigma_{\mathrm{n}}=$ mean background noise

However, there were limitations to this work. The analysis was performed on chest films that were converted to digital format, rather than digital images and only four line profiles were plotted. This was unable to capture the features of the entire lesion, gave only limited information on the distribution of background noise and introduced losses in the digitisation process, It was also dependent on the operators' choice of placement of the lines. A further limitation of the work was the decision to use the maximum angle from the four profiles rather than the mean value of the edge angles. This gave little insight into the radial extent of the maximum edge angle and could therefore overestimate its influence on the salience of the whole lesion. Nevertheless it showed there was value in making such measurement because it could help discriminate detection and decision errors, provide a perceptual guide to the technology of CAD and image enhancement processes and it extended the range of parameters available in eye-tracking experiments. The present work builds on this original set of ideas and provides a more refined and precise means of quantifying the perceptual salience of focal image features.

\section{Perceptual Measures}

Kundel et al [23] defined three lesion detection error classifications; search or scanning errors, recognition errors, and decision making errors. Scanning errors are due to the failure of the observer to fixate on the lesion, recognition errors are when the lesion is fixated on yet failing to detect it as a lesion, and decision making errors are incorrect interpretation of the observed lesion[23], by far the largest proportion of errors have found to be in decision making[23]. Other perceptual errors come from satisfaction of search (SOS) error, where the observers' attention is diverted from the lesion by a more conspicuous finding [24-26].

Perception based image quality assessment is far more useful for determining diagnostic capabilities than objective measures, as it is directly related to the way the operator looks at the images, where the quality is directly related to the ability to detect and recognise the lesion[27]. However human observer studies such as ROC-AUC[28] or 2AFC[29, 30] studies can be time consuming, and potentially expensive, and can also yield inconclusive findings, and so additional objective measures are useful to support human observer studies[27].

When Revesz et al[3] measured the conspicuity of lung nodules, and film readers were asked to determine the presence and location of any lesions, they found that the calculated conspicuity correlated with the probability of detection within 
a limited range of conspicuity values. Samei et al[5] measured the contrast-diameter product based on local anatomical noise, and found a correlation with detectability of the lesions with this measure.

However, attempting to correlate objective measures with human observer studies has not always proved successful, Manning et al[22] found poor correlation between the measure of conspicuity index and missed lesions in chest radiography and indicated that decision errors were more common than those of detection. However it is recognised that the approach to calculating conspicuity was limited, as only four profiles were applied and also the lack of correlation involved other types of observer error as originally noted by Kundel et al[22]. Mello-Thomas et al[31] also found during an eye tracking study, that unreported lesions often received adequate visual attention and other eye tracking studies have found that the conspicuity of a lesion, or the amount of time it is observed, is not the only reason they are not reported[22, 32-34]. In short, although errors in radiology are not confined to readers missing features because they are poorly demonstrated it is valuable to inform efforts to improve the radiological task with measures of image quality that use functional data on visual performance. Conspcuity indices make a contribution to this endeavour.

\section{METHODOLOGY}

\section{Software design}

A novel software programme was developed to enable analysis of focal lesions within DICOM images. Using a JAVA based programme, an operator is enabled to draw a region of interest (ROI) around a lesion. Any number of ROIs within the image can be drawn.

The software then plots 180 line profiles around the ROI, giving data $360^{\circ}$ around the lesion.

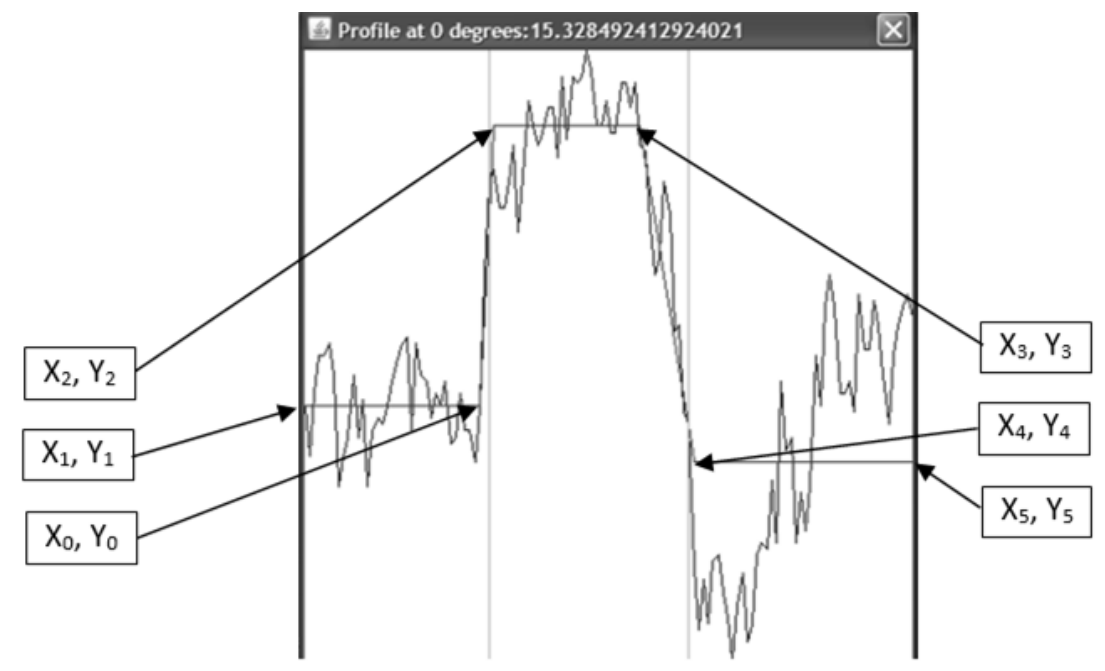

Figure 1 line profiles from ROI

Figure 1 gives an example of a single line profile across an ROI. The least squared method is used to fit the profile, this improves objectivity if the ROI is not drawn exactly around the lesion.

The following data is then exported as a TSV file for use in Excel; the profile values (in grey scale values) of $\mathrm{X}_{0,1,2,3,4 \& 5}$ $\mathrm{Y}_{0,1,2,3,4 \& 5}$, the standard deviations of the $\mathrm{Y}$ values between $\mathrm{X}_{0} \& \mathrm{X}_{1}\left(s d_{1}\right)$ and $\mathrm{X}_{4} \& \mathrm{X}_{5}\left(s d_{2}\right)$ are used to represent the (structural) background noise, and the standard deviation of the $\mathrm{Y}$ values between $\mathrm{X}_{2} \& \mathrm{X}_{3}$ (sdTop) are used to represent the noise within the lesion.

\section{Data analysis}

An Excel spreadsheet has been developed to import the TSV files and then to calculate the following: 


$$
\bar{d}=\overline{(X 3-X 2)}=\text { average lesion dimension }
$$

Equation 2

$$
\bar{\theta}=\overline{\tan ^{-1}\left[\frac{Y_{2}-Y_{1}}{X_{2}-X_{1}}\right], \tan ^{-1}\left[\frac{Y_{4}-Y_{3}}{X_{4}-X_{3}}\right]}=\text { average slope angle of all the line profiles }
$$

Equation 3

$\overline{\Delta G L}=\overline{Y_{2}-\frac{\left(Y_{1}+Y_{4}\right)}{2}}=$ the average difference in grey levels between the lesion and the background

Equation 4

$\overline{\sigma_{s}}=s d T o p$

Equation 5

$$
\overline{\sigma_{n}}=\frac{s d 1+s d 2}{2}
$$

Equation 6

This data was then used to calculate the Conspicuity Index $(\chi)$ :

$$
\chi=\frac{\bar{d} \tan [\bar{\theta}-1] \overline{\Delta G L}}{\sqrt{\overline{\sigma_{s}^{2}}+\overline{\sigma_{n}^{2}}}}
$$

Equation 7

A distinction between Equation 1 and Equation 7 is that instead of using the maximum slope angle, the mean value of slope and lesion dimension has been used, this is due to the sensitivity of the data, and the maximum values having the possibility of representing noise instead of real values. However, this can be amended to calculate any values as required.

CNR and SNR can also be calculated using the following equations

$$
\begin{aligned}
& \text { CNR }=\frac{\overline{Y_{2}-\left(\frac{Y_{1}+Y_{4}}{2}\right)}}{Y_{2}} \\
& S N R=\frac{\overline{Y_{2}-\left(\frac{Y_{1}+Y_{4}}{2}\right)}}{\sqrt{\overline{s d T o p^{2}}+\left(\overline{\frac{s d 1}{2}+\overline{s d 2}}\right)^{2}}}
\end{aligned}
$$

Equation 9

\section{Software reliability}

The software was tested for reliability by using the Gammex ACR CT accreditation phantom. A Toshiba Aquillion 16 CT scanner was used, and images were acquired using the following parameters: $\mathrm{kVp} 80,100,120,135 ; 50-400 \mathrm{mAs}$, in $50 \mathrm{mAs}$ increments; 1, 2, 3, 4 mm slice thickness (acquired and reconstructed) all using singe slices through Section 4 of 
the phantom, an example image is shown in Figure 2 below. ROIs were drawn and the conspicuity index was calculated. This was repeated four times to assure reproducibility of the conspicuity index calculation.

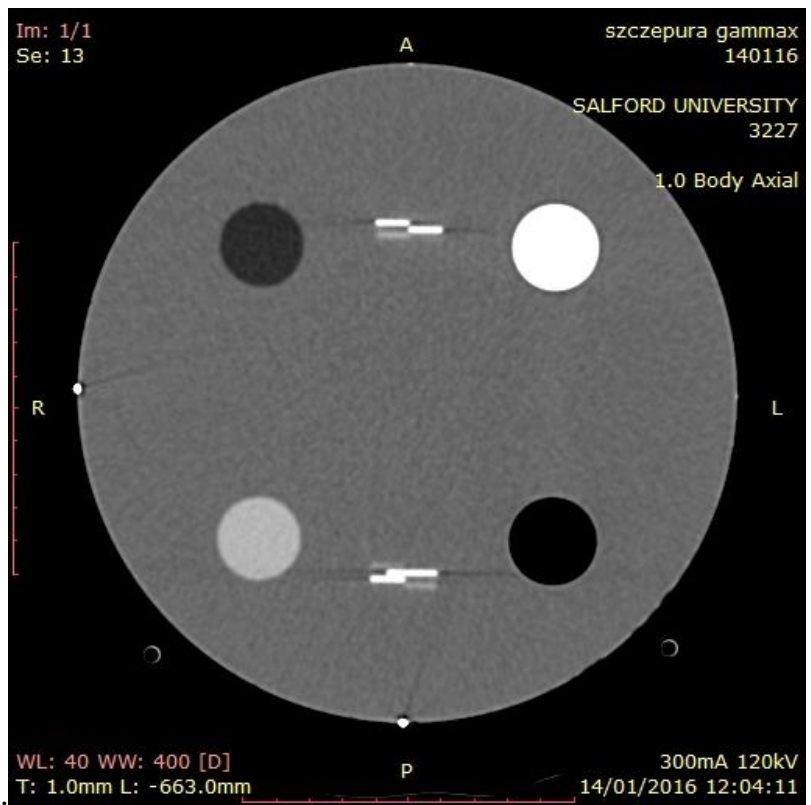

\section{Figure 2 - Gammex ACR CT Phantom}

\section{Phantom Data}

The software was also tested using a realistic anthropomorphic chest phantom "Lungman" [35], with 4 lesions inserted Figure 3, Figure 4 and Table 1 below.

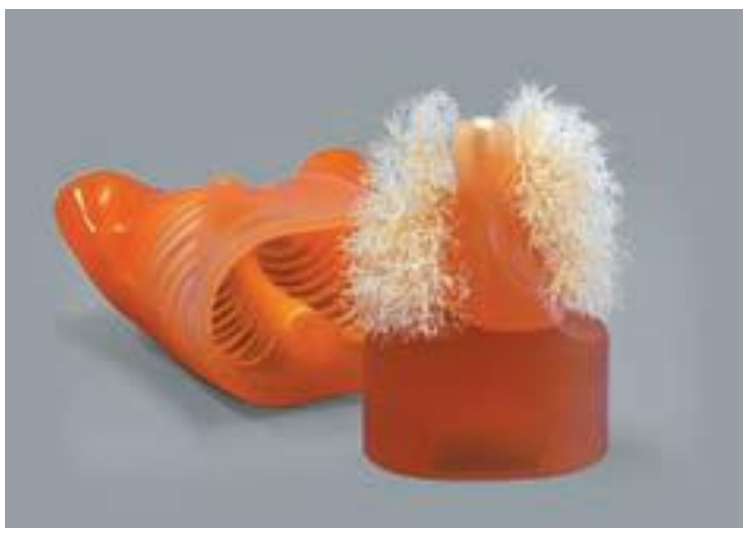

Figure 3 - Lungman phantom 


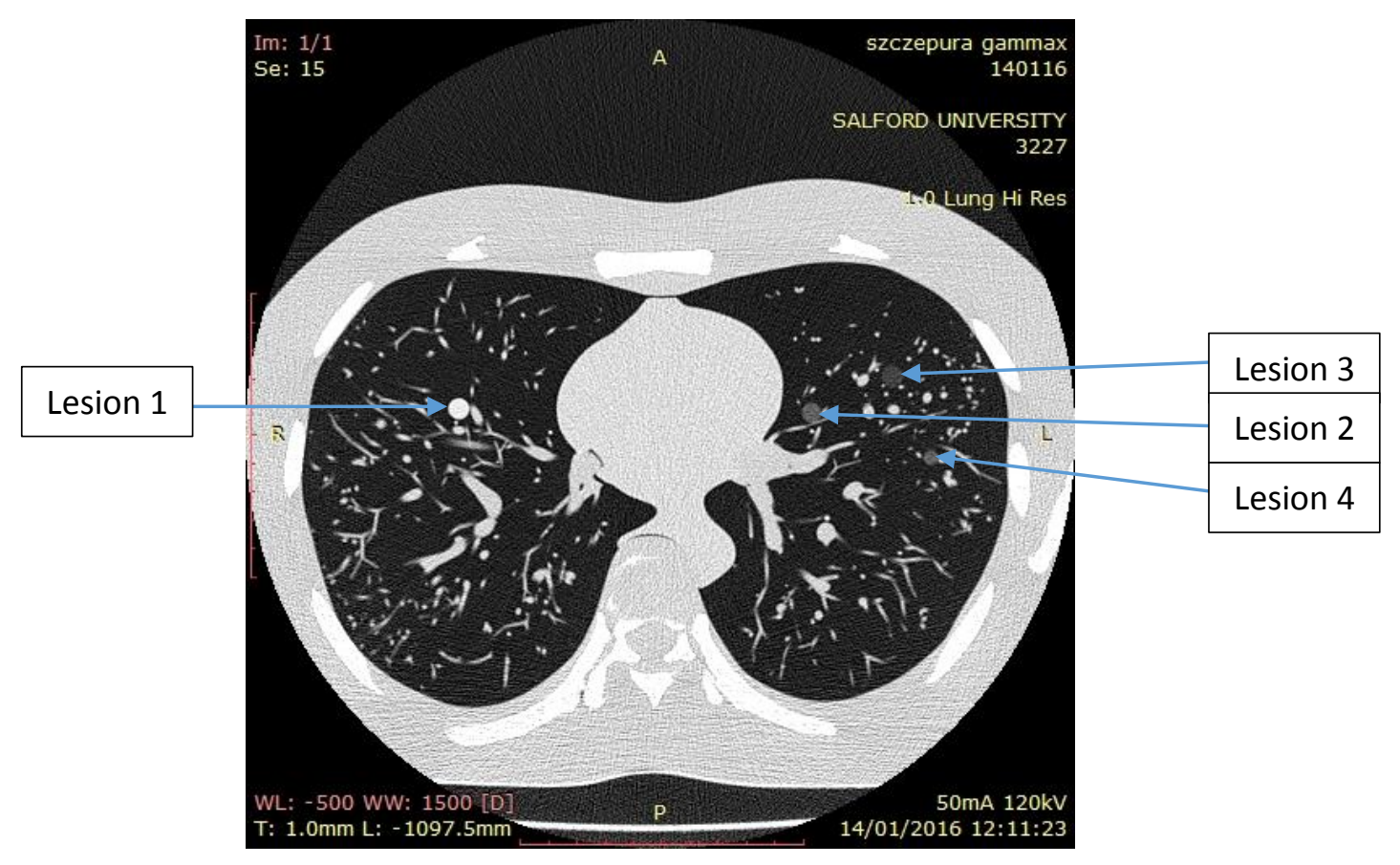

Figure 4 - CT image of Lungman phantom with lesions

\begin{tabular}{|c|c|c|}
\hline $\begin{array}{c}\text { Lesion } \\
\text { Number }\end{array}$ & Size (mm) & Hounsfield Units \\
\hline 1 & 8 & +100 \\
\hline 2 & 8 & -630 \\
\hline 3 & 8 & -800 \\
\hline 4 & 5 & -800 \\
\hline
\end{tabular}

\section{Table 1 - Lesion inserted into Lungman phantom}

The phantom was imaged using the same CT scanner on a $1 \mathrm{~mm}$ High Resolution Chest CT protocol, with varying mAs of between 25-600 mAs.

\section{RESULTS}

Software reliability 
Conspicuity Index $1 \mathrm{~mm} \mathrm{ST}$

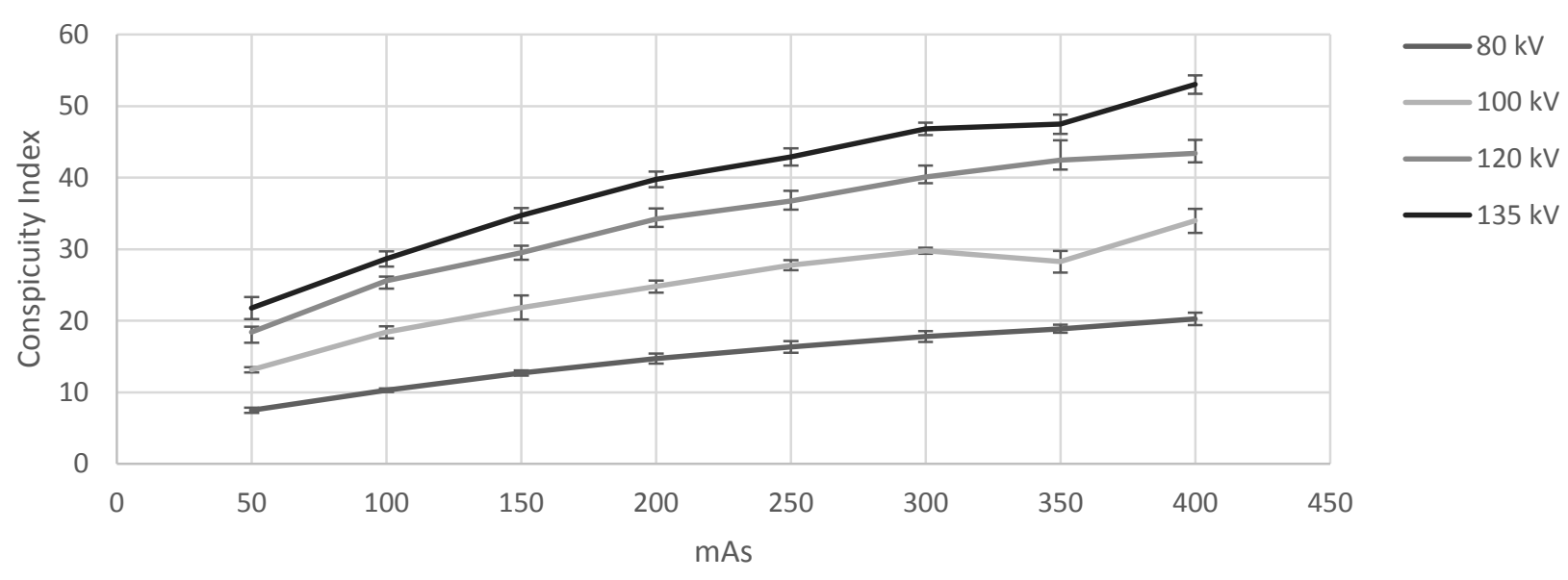

\section{Graph 1 Conspicuity Index - 1mm Slice Thickness}

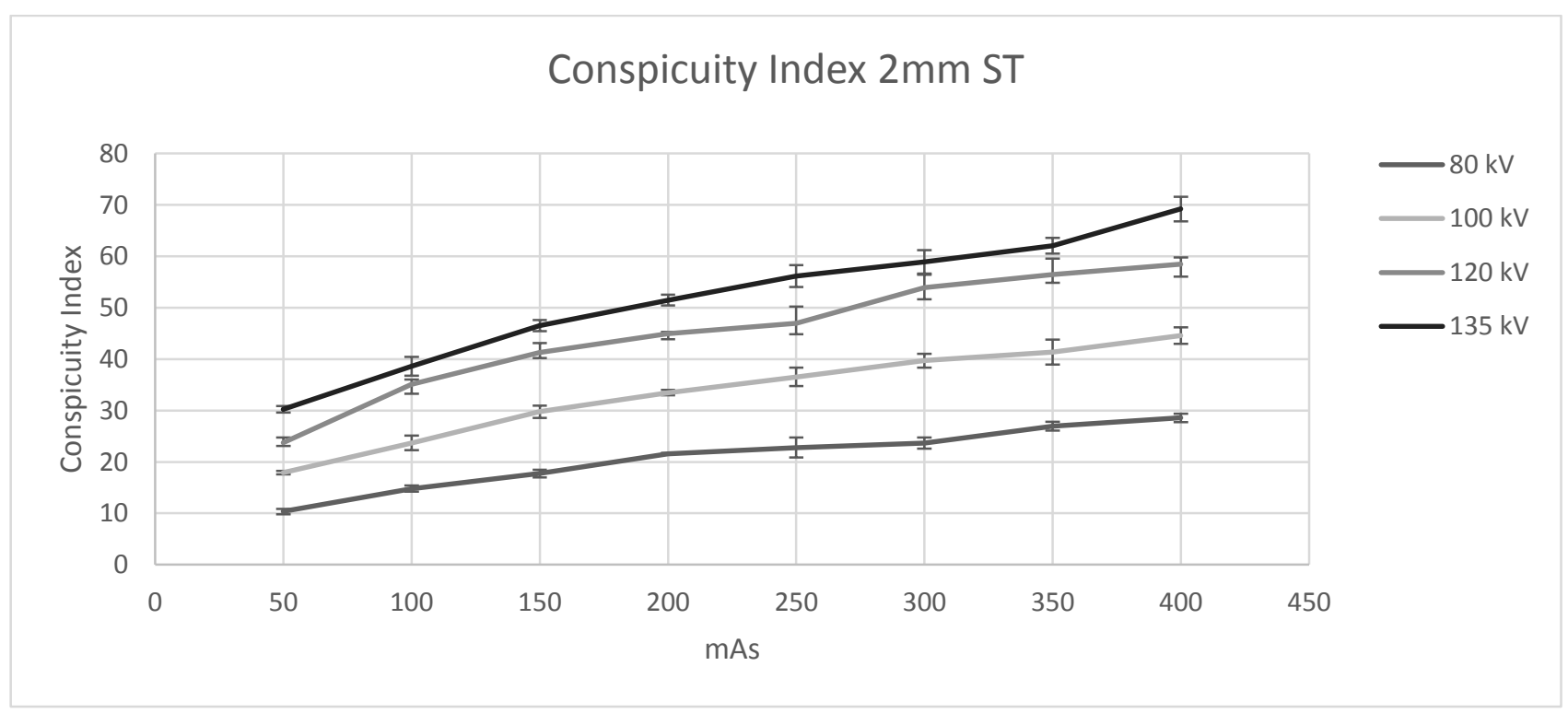

Graph 2 Conspicuity Index - 2mm Slice Thickness 
Conspicuity Index 3mm ST

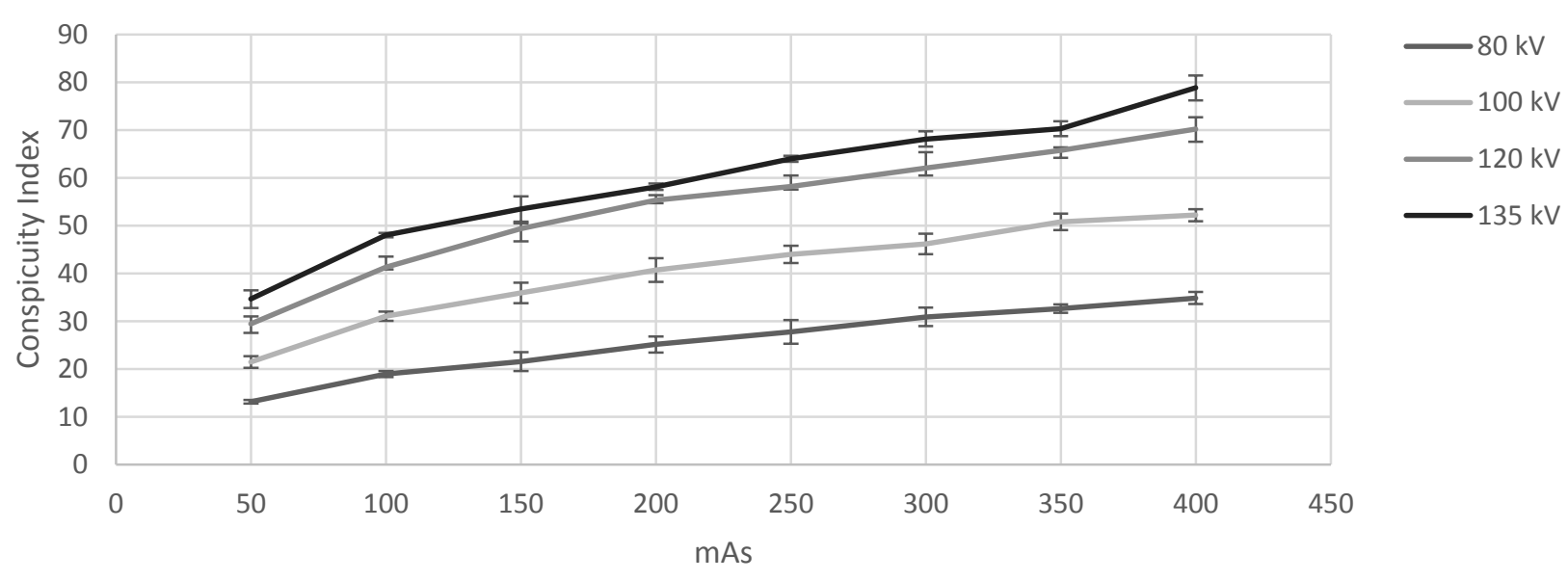

\section{Graph 3 Conspicuity Index - 3mm Slice Thickness}

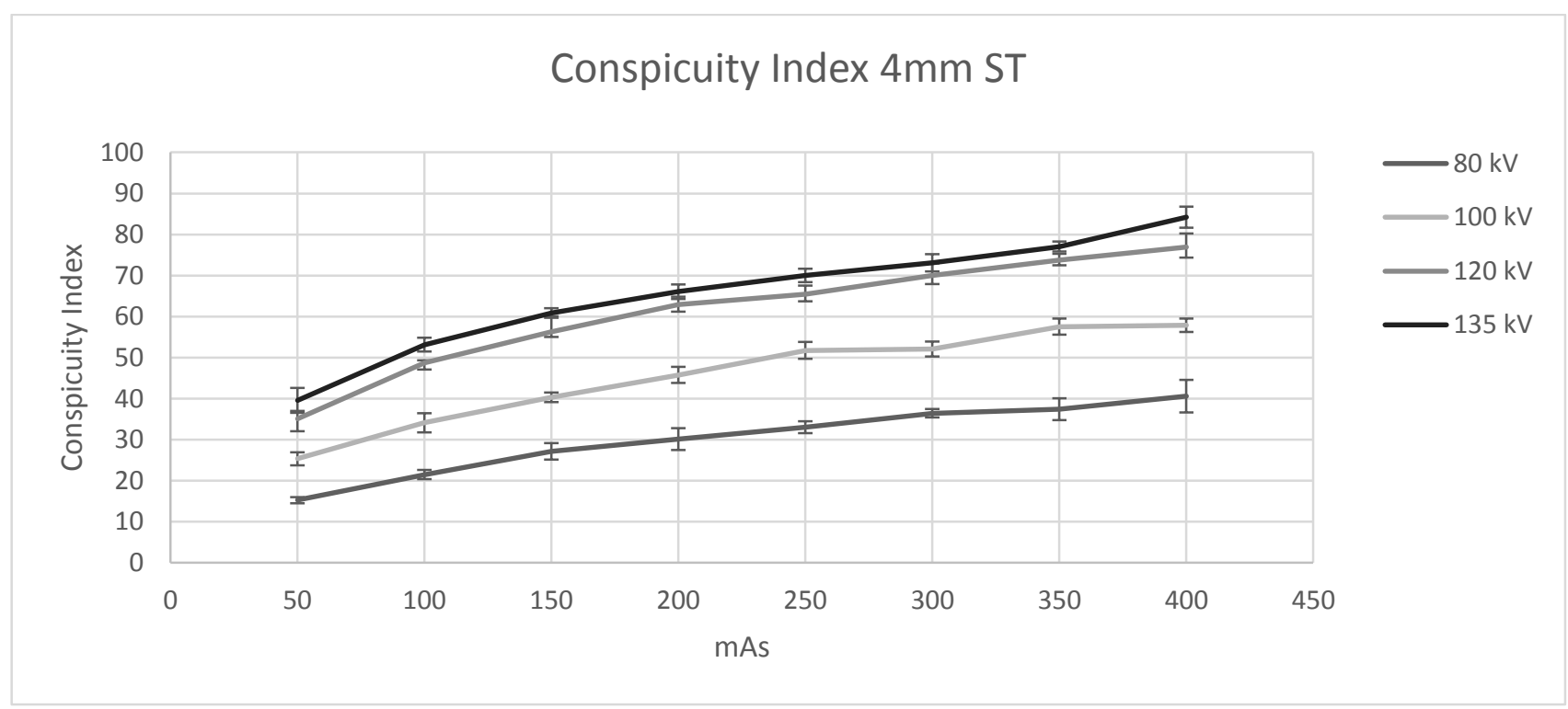

Graph 4 Conspicuity Index - $4 \mathrm{~mm}$ Slice Thickness 
Phantom Data

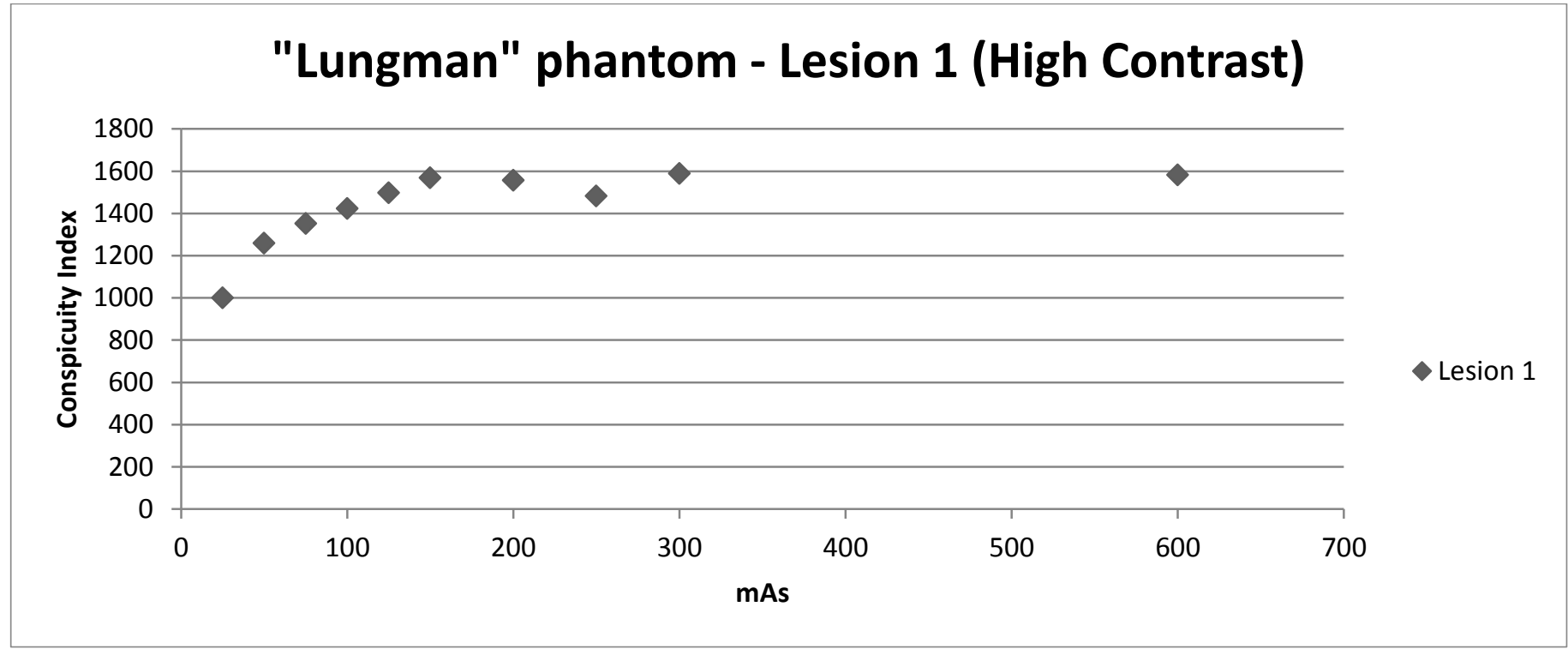

Graph 5 - "Lungman" phantom lesion 1

\section{"Lungman" phantom - low contrast lesions}

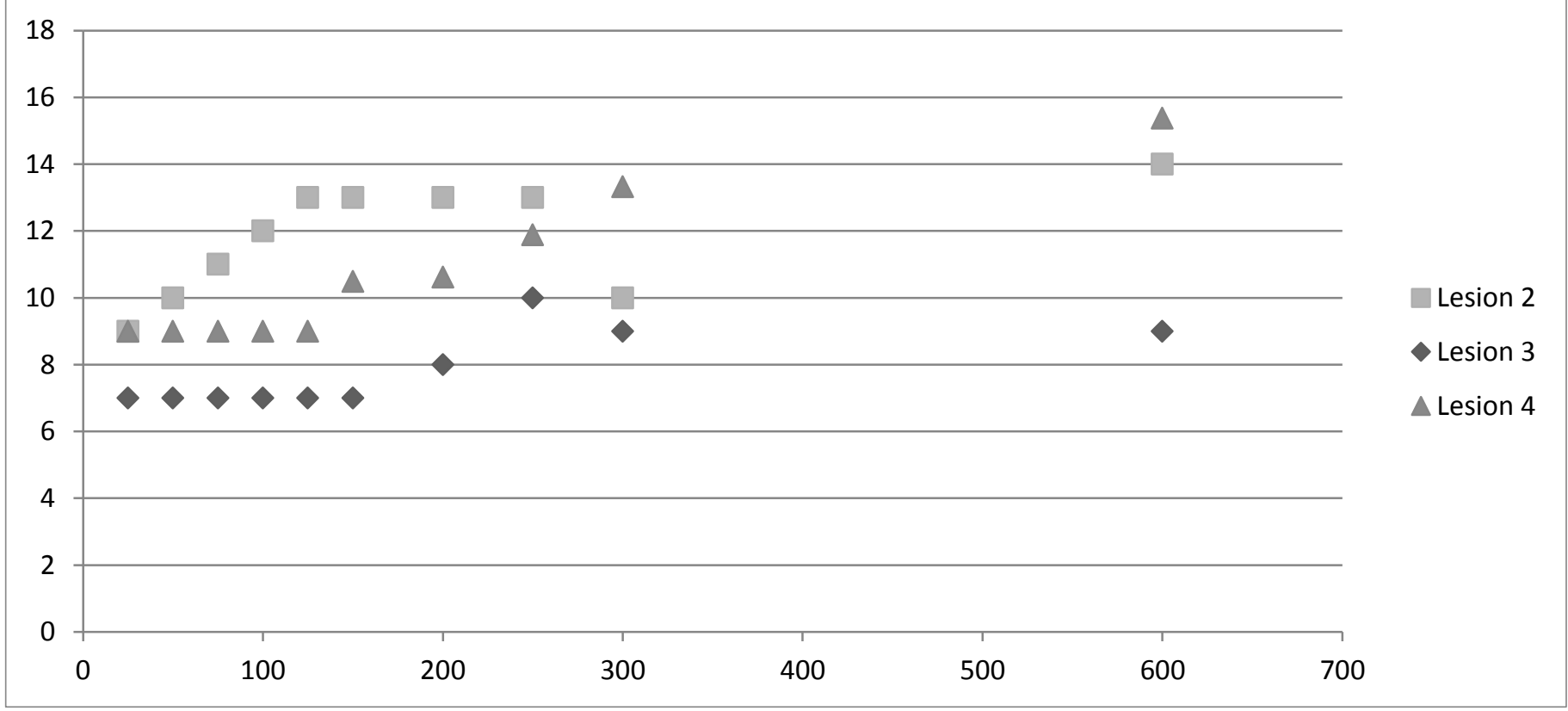

Graph 6 - "Lungman" conspicuity index- low contrast lesions 


\section{DISCUSSION}

All reliability results (Graph 1, Graph 2, Graph 3, Graph 4) were linear with mAs and behaved as expected with varying $\mathrm{kVp}$ and slice thickness. This demonstrates that the software is reliable in terms of representing the conspicuity within a DICOM image. Also, the ROIs were drawn 4 times, and as can be seen from these graphs, the standard deviations are very low, demonstrating reproducibility.

The more clinically representative phantom data using the "Lungman" phantom also demonstrates the reliability of the software, as even in a more complex phantom the results were still as expected with the changing parameters. Graph 5 demonstrates that the conspicuity index increases with $\mathrm{mAs}$ up to a point where it plateaus. This demonstrates the lack of improvement in conspicuity with increased $\mathrm{mAs}$, and demonstrates the capability of the software to provide optimisation opportunities for clinical imaging. Graph 6 demonstrates that with low contrast lesions, the conspicuity is low, as expected, the lesions with a greater difference to background (lesion 3) shows the highest conspicuity index, with lesion 4 being the lowest value, as this is the smallest lesion $(5 \mathrm{~mm})$

The software has been used on real clinical images, and been shown to function with DICOM images from various modalities and manufacturers, however it has not yet been used to assess the diagnostic performance of real clinical images. Due to the radiation risk, the reliability of the software cannot be established using real clinical images as variables would have to be repeatedly changed on the same participant to demonstrate the behaviour of the conspicuity index, and this would require ethical approval.

Now the software and calculations have been found to be reliable, future work with this software is to compare to perceptional analysis, such as JAFROC, 2AFC and eye tracking.

Although correlations of these distinct datasets may be possible in simple controlled images, such as physics phantoms, there will be the added complications of decision errors, and so this software has the potential to distinguish between perceptual and cognitive errors.

Currently this research has focused on focal lesions, however it is recognised that there are many other lesion types, such as linear or diffuse, future developments of the software may include analysis of these types of lesions.

\section{CONCLUSION}

A novel software has been developed that enables the calculation of the conspicuity index of focal lesions in DICOM images.

The software and associated Excel spreadsheet have been tested for reliability and has been shown to provide reliable reproducible results.

The analysis could be further developed to incorporate JAFROC and eye-tracking data allowing correlations between physical and perception measures to be made beyond basic CNR calculations, it could also be used as a tool to distinguish between perceptual and cognitive error.

Further refinements could lead to measures of the detectability of more diffuse disease features.

[1] E. Samei, "Performance of Digital Radiographic Detectors: Quantification and Assessment Methods." 33-37.

[2] J. T. Bushberg, J. A. Seibert, E. M. Leidholdt et al., [The essential physics of medical imaging] Wolters Kluwer/Lippincott Williams \& Wilkins, Philadelphia, Pa. ; London(2012). 
G. Revesz, H. L. Kundel, and M. A. Graber, "The influence of structured noise on the detection of radiologic abnormalities," Invest Radiol, 9(6), 479-86 (1974).

[4] E. E. Coche, B. Ghaye, J. Mey et al., [Comparative Interpretation of CT and Standard Radiography of the Chest [electronic resource]] Springer Berlin Heidelberg, Berlin, Heidelberg(2011).

[5] E. Samei, M. J. Flynn, E. Peterson et al., "Subtle lung nodules: influence of local anatomic variations on detection," Radiology, 228(1), 76-84 (2003).

[6] F. Li, S. Sone, H. Abe et al., "Lung cancers missed at low-dose helical CT screening in a general population: comparison of clinical, histopathologic, and imaging findings," Radiology, 225(3), 673-83 (2002).

[7] U. Plöckinger, "Diagnosis and Treatment of Gastrinomas in Multiple Endocrine Neoplasia Type 1 (MEN-1)," Cancers (Basel), 4(1), 39-54 (2012).

[8] R. L. Birdwell, D. M. Ikeda, K. F. O'Shaughnessy et al., "Mammographic characteristics of 115 missed cancers later detected with screening mammography and the potential utility of computer-aided detection," Radiology, 219(1), 192-202 (2001).

[9] J. Michaelson, S. Satija, R. Moore et al., "Estimates of the Sizes at Which Breast Cancers Become Detectable on Mammographic and Clinical Grounds," Journal of Women's Imaging, 5(1), 3-10 (2003).

[10] S. C. Bushong, [Radiologic science for technologists : physics, biology, and protection] Elsevier Mosby, St. Louis, Mo. ; [London](2008).

[11] A. Burgess, [Spatial vision research without noise] Cambridge University Press, Cambridge, 3 (2010).

[12] V. F. van Ravesteijn, T. N. Boellaard, M. P. van der Paardt et al., "Electronic Cleansing for 24-H Limited Bowel Preparation CT Colonography Using Principal Curvature Flow," IEEE Trans Biomed Eng, 60(11), 303645 (2013).

[13] K. W. Yeom, S. J. Holdsworth, A. T. Van et al., "Comparison of readout-segmented echo-planar imaging (EPI) and single-shot EPI in clinical application of diffusion-weighted imaging of the pediatric brain," AJR Am J Roentgenol, 200(5), W437-43 (2013).

[14] L. I. Peltonen, A. A. Aarnisalo, M. K. Kortesniemi et al., "Limited cone-beam computed tomography imaging of the middle ear: a comparison with multislice helical computed tomography," Acta Radiol, 48(2), 207-12 (2007).

[15] M. P. Botelho, R. Agrawal, F. D. Gonzalez-Guindalini et al., "Effect of radiation dose and iterative reconstruction on lung lesion conspicuity at MDCT: does one size fit all?,” Eur J Radiol, 82(11), e726-33 (2013).

[16] R. T. Gupta, C. M. Iseman, J. R. Leyendecker et al., "Diagnosis of focal nodular hyperplasia with MRI: multicenter retrospective study comparing gadobenate dimeglumine to gadoxetate disodium," AJR Am J Roentgenol, 199(1), 35-43 (2012).

[17] M. L. Zuley, K. M. Willison, E. Bonaccio et al., "Full-field digital mammography on LCD versus CRT monitors," AJR Am J Roentgenol, 187(6), 1492-8 (2006).

[18] D. Marin, K. R. Choudhury, R. T. Gupta et al., "Clinical impact of an adaptive statistical iterative reconstruction algorithm for detection of hypervascular liver tumours using a low tube voltage, high tube current MDCT technique," Eur Radiol, 23(12), 3325-35 (2013).

[19] I. B. Weiner, and W. E. Craighead, [The Corsini encyclopedia of psychology] John Wiley, Hoboken, N.J.(2010).

[20] O. R. Brook, S. Gourtsoyianni, A. Brook et al., "Split-Bolus Spectral Multidetector CT of the Pancreas: Assessment of Radiation Dose and Tumor Conspicuity," Radiology, (2013).

[21] W. Chang, J. M. Lee, K. Lee et al., "Assessment of a model-based, iterative reconstruction algorithm (MBIR) regarding image quality and dose reduction in liver computed tomography," Invest Radiol, 48(8), 598-606 (2013).

[22] D. J. Manning, S. C. Ethell, and T. Donovan, "Detection or decision errors? Missed lung cancer from the posteroanterior chest radiograph,” Br J Radiol, 77(915), 231-5 (2004).

[23] H. L. Kundel, C. F. Nodine, and D. Carmody, "Visual scanning, pattern recognition and decision-making in pulmonary nodule detection," Invest Radiol, 13(3), 175-81 (1978).

[24] K. S. Berbaum, E. A. Franken, D. D. Dorfman et al., "Role of faulty decision making in the satisfaction of search effect in chest radiography," Acad Radiol, 7(12), 1098-106 (2000).

[25] K. S. Berbaum, E. A. Brandser, E. A. Franken et al., "Gaze dwell times on acute trauma injuries missed because of satisfaction of search," Acad Radiol, 8(4), 304-14 (2001). 
[26] K. S. Berbaum, D. D. Dorfman, E. A. Franken et al., "Proper ROC analysis and joint ROC analysis of the satisfaction of search effect in chest radiology," Acad Radiol, 7(11), 945-58 (2000).

[27] G. B, L. Q, P. L et al., "Objectively measuring signal detectability, contrast, blur and noise in medical images using channelized joint observers." 8673.

[28] J. D. Thompson, D. J. Manning, and P. Hogg, "The value of observer performance studies in dose optimization: a focus on free-response receiver operating characteristic methods," J Nucl Med Technol, 41(2), 57-64 (2013).

[29] A. E. Burgess, "Comparison of receiver operating characteristic and forced choice observer performance measurement methods,” Med Phys, 22(5), 643-55 (1995).

[30] S. Richard, and J. H. Siewerdsen, "Comparison of model and human observer performance for detection and discrimination tasks using dual-energy x-ray images," Med Phys, 35(11), 5043-53 (2008).

[31] C. Mello-Thoms, L. Hardesty, J. Sumkin et al., "Effects of lesion conspicuity on visual search in mammogram reading," Acad Radiol, 12(7), 830-40 (2005).

[32] C. Mello-Thoms, "The problem of image interpretation in mammography: effects of lesion conspicuity on the visual search strategy of radiologists," Br J Radiol, 79 Spec No 2, S111-6 (2006).

[33] D. S. Brettle, E. Berry, and M. A. Smith, "The effect of experience on detectability in local area anatomical noise," Br J Radiol, 80(951), 186-93 (2007).

[34] M. S. Chesters, "Human visual perception and ROC methodology in medical imaging," Phys Med Biol, 37(7), 1433-76 (1992).

[35] Kyotokagaku, [Multipurpose Chest Phantom N1 "Lungman"]. 